\title{
Injection laryngoplasty treatment for vocal atrophy in the elderly
}

\begin{abstract}
Aging produces physiological changes that can alter the voice, vocal fold atrophy being the most frequent, producing communication difficulties and problems with selfimage. Since the elderly population is the fastest growing segment of the population, and it is staying in the workforce longer, more patients are seeking help for their voice complaints. Among different treatment options, percutaneous injection laryngoplasty with hyaluronic acid is a safe and effective procedure in the management of dysphonia due to age-related vocal fold atrophy.
\end{abstract}

Keywords: vocal fold atrophy, injection laryngoplasty, dysphonia
Volume 2 Issue 2 - 2017

\author{
María José Orellana, Pedro Badía, Carla \\ Napolitano \\ Department of Otorhinolaryngology, Universidad Católica de \\ Chile, Chile
}

Correspondence: María José Orellana, Department of Otorhinolaryngology, Universidad Católica de Chile, Chile, Tel +56993593942, Email cnapolitanov@gmail.com

Received: July 29, 2017 | Published: September 06, 2017

\section{Objective}

Divulge injection laryngoscopy as a treatment for dysphonia secondary to vocal fold atrophy in the elderly.

\section{Introduction}

Elderly patients present deterioration in voice quality due to anatomical and functional changes of the larynx associated with the aging process, and other voice disorders common in the elderly population. Identified causes of geriatric voice dysfunction include anatomic changes in the larynx such as vocal fold atrophy, central neurologic conditions (e.g. stroke, Parkinson's disease, essential tremor, spasmodic dysphonia), impaired physiologic conditioning (e.g. decreased lung elasticity and compliance, reduced physical fitness), and pathologic lesions such as laryngeal carcinoma, benign vocal fold lesions (e.g. epithelial lesions, Reinke's edema, cysts), inflammatory disorders (e.g., reflux laryngitis), infectious laryngitis (e.g., fungal laryngitis), vocal fold paralysis, and reflux laryngitis. ${ }^{1,2}$

The most common diagnosis in the setting of an older patient with a voice disorder is vocal fold atrophy associated with incomplete glottic closure, called presbylaryn $x^{3}$ which produces changes in voice quality including breathiness, weakness, tremulousness, hoarseness, inability to sustain phonation, and inadequate loudness level. ${ }^{1}$ These vocal changes can produce communication difficulties, deterioration of their quality of life and general performance, and also problems with their self-images, because the voice has a strong personal identity meaning. ${ }^{3}$

On the other hand, glottic closure is one of the airway protective mechanisms, so glottic insufficiency secondary to vocal fold atrophy is associated with the swallowing disorder of the elderly. ${ }^{3}$ The proportion of patients aged 65 and over has been increasing in the last decades, and the incidence of vocal fold atrophy identified in this group is about $20 \%$. This accounts for greater than 10 million people in the United States per the US Census Bureau, and 33\% of this population were still in the workforce or participating in hobby groups of singing, poetry or another hobby involving the use of voice..$^{1,4,5}$ As the number of patients that might require improvement of their voice is increasing, medical treatment for this condition is taking more importance.
Treatment options for dysphonia due to vocal atrophy include: reassurance, voice therapy, injection laryngoplasty and medialization thyroplasty. ${ }^{2}$ Injection laryngoplasty consists in medialization of the vocal fold(s) by injecting a material into the thyroarytenoid muscle, favoring glottic closure, thus improving both phonation and swallowing function. ${ }^{6}$

Temporary injection medialization can be performed under general anesthesia or in the awake-patient setting with local anesthesia, the latter being the one of choice especially in elderly patients since it avoids the need for general anesthesia, in addition to being able to immediately "regulate" the voice in relation to the amount of injected material. ${ }^{6}$ Injection laryngoplasty can be performed by different approaches: trans-oral, percutaneous and trans-nasal endoscopic.

There are different options of synthetic and biological materials that can be used: long-lasting mainly calcium hydroxyapatite (Radiesse Voice $^{\circledR}$ ) or temporary such as carboxymethylcellulose (Radiesse Voice $\mathrm{Gel}^{\circledR}$ ) or hyaluronic acid (Restylane ${ }^{\circledR}$ ). Of the temporary injection materials, one of the most popular is hyaluronic acid because it is resorbable, does not induce local inflammatory response and maintains the viscoelasticity of the superficial lamina propria of the vocal fold. ${ }^{6}$ Being a temporary material, it can be a "test injection" as it allows reversibility in case of unsatisfactory result, or the possibility of successive injections if the results were satisfactory. It also can be followed by a later injection with a longer duration material or a later definitive treatment such as medialization thyroplasty. ${ }^{6}$

\section{Case report}

We present the case of a 84 year-old man with history of a compensated left vocal fold paralysis secondary to a total thyroidectomy 30 years ago, who consulted for a 2 year progressive dysphonia. He has a Voice-related quality of life (V-RQOL) score of 32 and a poor voice self perception (V-RQOL is a standardized questionnaire that measures the impact that the voice disorder is having on the patient's quality of life; the score ranges from 10 -absence of symptoms- to 50 -worst symptoms possible-). ${ }^{7}$

Laryngeal videostroboscopy evidenced a compensated former vocal fold paralysis and a bilateral vocal fold atrophy with a secondary glottic insufficiency, the latter causing the current voice complaints (Figure 1). Voice therapy was carried out without significant 
improvement, so a percutaneous bilateral medialization injection laryngoplasty with Hyaluronic Acid (Restylane ${ }^{\circledR}$ ) was indicated. The procedure was well tolerated, no complications were reported, and the patient had a favourable immediate vocal improvement (Figure 2 ). At the evaluation 8 days after the procedure the patient referred a significant improvement in voice quality and volume, a V-RQOL score of 12 and a good voice self perception (Figure 3). (lamentably, we do not have a longer follow up).

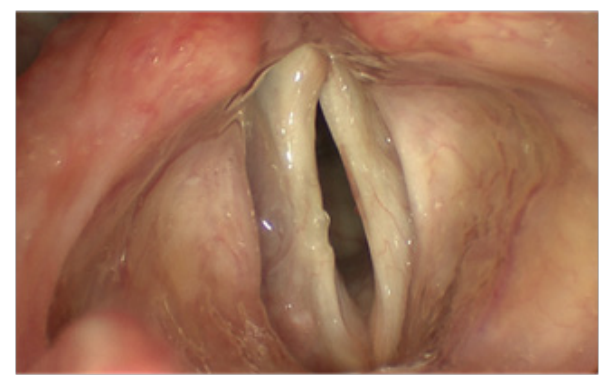

Figure I Incomplete glottal closure due to left vocal fold paralysis and bilateral vocal fold atrophy, with vocal fold bowing, causing glottic insufficiency.

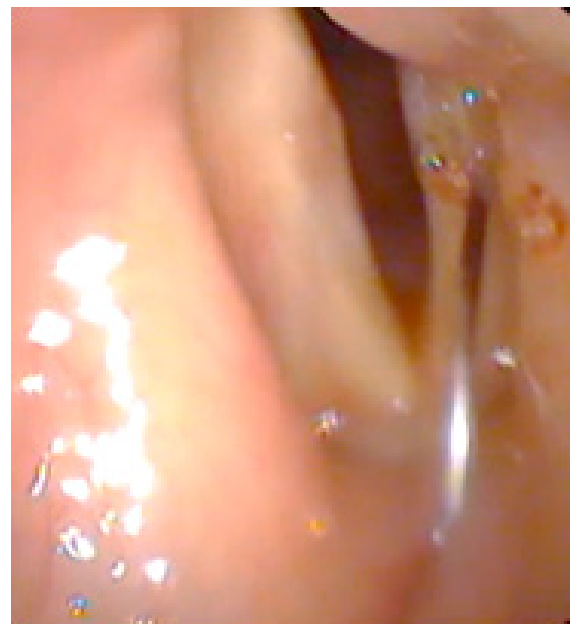

Figure 2 Nasofibroscopic view of a percutaneous injection laryngoplasty, the needle injects hyaluronic acid into the thyroarytenoid muscle of the vocal fold.

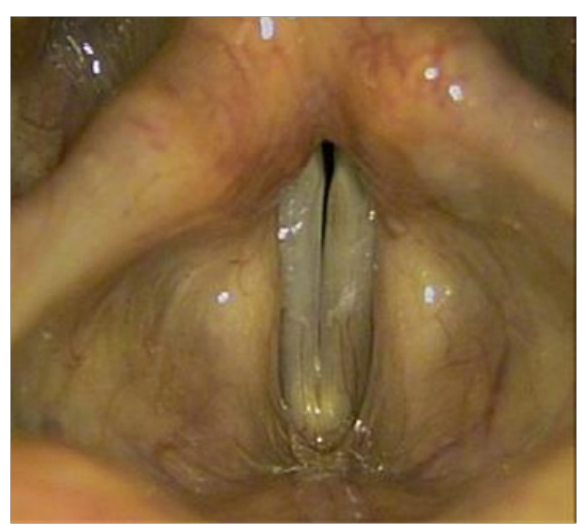

Figure 3 Laryngeal videostroboscopy showing complete glottic closure 8 days after the injection.

\section{Discussion}

A retrospective study of 6360 patients seen over a 6-year period at the Emory Voice Center for otolaryngologic complaints showed that $21 \%$ were over the age of $65,775(58 \%)$ of which had vocal complaints, with the most common diagnosis being vocal fold atrophy $(25 \%)$. Of those patients diagnosed with vocal fold atrophy, the majority opted for voice therapy $(57 \%)$, followed by reassurance $(39 \%)$, and injection laryngoplasty $(6 \%)$, the latter with a statistically significant improvement in mean pre-therapy and post-therapy VRQOL score. ${ }^{8}$

While the elderly population is growing and there is an increase in the elderly people in the workforce, there is a noted need for treatment options for voice complaints in this population. Injection laryngoplasty is a procedure that can be performed in the awakepatient setting, used to treat glottic insufficiency, and has been used successfully in age-related vocal fold atrophy. Injection laryngoplasty is a safe procedure with a low rate of complications: vasovagal reactions, vocal fold hemorrhage $(1,2 \%)$, misplaced injection material $(5 \%)$, early resorption of injected material requiring a second injection (3.6\%), and very infrequent adverse reactions to hyaluronic acid. ${ }^{6}$ The rate of procedure suspension reported is $5,7 \%{ }^{9}$ because of pain or anxiety, cough or poor laryngeal visualization.

Percutaneous injection laryngoplasty with hyaluronic acid can be recommended as a well tolerated, safe and effective procedure in the management of dysphonia secondary to age-related vocal fold atrophy, allowing significant improvement in social and work quality of life in the elderly.

\section{Acknowledgements}

Patient consent related case report take the permission from concerned patient.

\section{Conflict of interest}

Author declares there is no conflict of interest in publishing this manuscript.

\section{References}

1. Takano S, Kimura M, Nito T, et al. Clinical analysis of presbylarynxvocal fold atrophy in elderly individuals. Auris Nasus Larynx. 2010;37(4):461-464.

2. Davids T, Klein AM, Johns MM. Current dysphonia trends in patients over the age of 65: is vocal atrophy becoming more prevalent? The Laryngoscope. 2012;122(2):332-335.

3. Pontes P, Brasolotto A, Behlau M. Glottic characteristics and voice complaint in the elderly. $J$ Voice. 2005;19(1):84-94.

4. Lundy DS, Silva C, Casiano RR, et al. Cause of hoarseness in elderly patients. Otolaryngol Head Neck Surg. 1998;118(4):481-485.

5. Patel AK, Mildenhall NR, Kim W, et al. Symptom overlap between laryngopharyngeal reflux and glottic insufficiency in vocal fold atrophy patients. Ann Otol Rhinol Laryngol. 2014;123(4):265-270.

6. Badía P, Winter M, León $\mathrm{N}$, et al. Laringoplastía de inyección con ácido hialurónico, experiencia del Hospital Clínico de la Pontificia Universidad Católica de Chile. Revista de otorrinolaringología y cirugía de cabeza y cuello. 2015;75(3):232-238.

7. Hogikyan ND, Sethuraman G. Validation of an instrument to measure voice-related quality of life (V-RQOL). J Voice. 1999;13(4):557-569.

8. Verdonck LIM, Mahieu HF. Vocal aging and the impact on daily life: a longitudinal study. Journal of Voice. 2004;18(2):193-202.

9. Mathison CC, Villari CR, Klein AM, et al. Comparison of outcomes and complications between awake and asleep injection laryngoplasty: A Case-Control Study. Laryngoscope. 2009;119(7):1417-1423. 